\title{
石墨烯化聚合物：一种兼具电子和离子传输通道的 三维富碳高分子能源材料
}

\author{
梁家旭 肖志昌 智林杰 ${ }^{*}$ \\ (中国科学院纳米科学卓越创新中心, 国家纳米科学中心, 北京 100190; 中国科学院大学, 北京 100049)
}

\begin{abstract}
摘要: 近年来, 用于电化学能源存储和转化的石墨烯材料, 得到了研究者们越来越多的关注。但是, 这些石 墨烯材料不同于严格定义的单原子碳层结构, 往往具有孔洞、杂原子和化学官能团等缺陷结构。由于制备方 法的不同, 缺陷结构各不相同, 其电化学性能也表现各异。结构分析表明, 这类材料是由类似石墨烯片段的 单元与聚合物链共价连接而成, 使其具有石墨烯和聚合物的双重特性, 我们称之为石墨烯化聚合物。由小分 子通过自下而上的方法制备的多孔聚合物, 也可以通过进一步热交联等方法, 使其形成包含石墨烯片段单元 与聚合物链的化学结构。这些材料与石墨烯衍生材料一起组成了石墨烯化聚合物的整个谱系; 这个谱系涵盖 了由聚合物到石墨烯的过渡区。更重要的是, 这类材料特殊的结构与性质, 使其成为一种兼具电子和离子传 输通道的三维富碳高分子材料, 非常适合作为电极材料应用于电化学能源存储和转化, 这为我们深入研究储 能器件中电极材料的结构与性能的相关关系提供了很好的材料平台。
\end{abstract}

关键词: 石墨烯化聚合物; 富碳高分子; 能源材料; 电化学; 能源存储和转化 中图分类号: 0646

\section{Graphenal Polymers: 3D Carbon-Rich Polymers as Energy Materials with Electronic and lonic Transport Pathways}

\author{
LIANG Jia-Xu XIAO Zhi-Chang ZHI Lin-Jie* \\ (CAS Center of Excellence for Nanoscience, National Center for Nanoscience and Technology, Beijing 100190, P. R. China; \\ University of Chinese Academy of Sciences, Beijing 100049, P. R. China)
}

\begin{abstract}
Graphene and its derivatives have attracted increasing attention during the last decade as efficient materials for the storage and conversion of energy. In most cases, however, these graphene materials possess large numbers of structural defects such as cavities, heteroatoms and functional groups, making them quite different from the precisely-defined "single carbon layer of graphite" observed for graphene. These materials also differ considerably in terms of their electrochemical properties because of their variable structures, which are strongly influenced by the methods used during their preparation. Structural analyses have indicated that these materials consist of graphene subunits, which are interconnected by organic linkers with properties lying between those of graphene and polymers, which we have defined as "graphenal polymers". The thermal crosslinking reactions of porous polymer networks fabricated from small organic molecules using a bottom-up strategy also result in graphene-like subunits, which are covalently interconnected by polymeric fractions. These materials cover a series of transitional intermediates belonging to the "graphenal polymers" family, where polymers and graphene sit at opposite ends of family spectrum. Moreover, the special structures and properties of these materials make them ideal electrode materials for the storage and conversion of energy via electronic and ionic
\end{abstract}

Received: June 2, 2016; Revised: July 13, 2016; Published online: July 13, 2016.

*Corresponding author. Email: zhlj@nanoctr.cn; Tel: +86-10-82545578.

The project was supported by the Ministry of Science and Technology of China (2012CB933403) and National Natural Science Foundation for Distinguished Young Scholars, China (51425302).

科技部纳米专项课题(2012CB933403)和国家自然科学基金委杰出青年基金(51425302)资助

(c) Editorial office of Acta Physico-Chimica Sinica 
transport pathways, allowing for a deeper evaluation of the structure-property relationships of different electrode materials.

Key Words: Graphenal polymer; Carbon-rich polymer; Energy material; Electrochemistry; Energy storage and conversion

\section{1 引言}

在现代生活中, 环境污染和传统能源枯竭等 问题变得越来越突出，低成本、环境友好、高性 能的电化学能源存储与转化器件越来越引起国际 社会的广泛关注。目前研究最多的电化学能源存 储和转化体系包括锂离子电池、燃料电池和超级 电容器。这些器件虽然在能源存储和转化上的具 体机理各不相同, 但是它们具有电化学上的相似 性, 即所涉及的电化学反应均在电极材料和电解 液的界面上进行, 器件的性能很大程度上取决于 电极材料的结构特性, 其中主要参数包括电极材 料的电导率、电极材料的多孔特性、化学反应位 点的分布、电解液的电导率、以及反应物和产物 的扩散性能等。这就需要电极材料同时具有优异 的电子导通和离子传输性能, 并且在亚纳米甚至 分子尺度上具有精确的结构 ${ }^{1}$ 。

石墨烯材料由于具有超高的电子迁移率、优 异的机械性能、良好的导热性能以及巨大的比表 面积等特性, 得到了国内外学术界的高度重视, 目前已成为电化学领域研究的热点材料 ${ }^{2}$ 。石墨烯

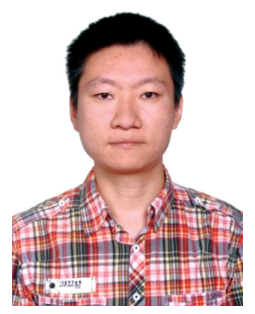

梁家旭, 1986 年生。分别于 2008 年和 2012 年在北京理工大学获学士 和硕士学位。2014年至今, 在国家 纳米科学中心攻读博士学位。主要 研究方向为富碳纳米材料的制备和 表征及其在能源环境领域的应用。
最初是由高定向石墨经过机械剥离制备而成, 这 种方法得到的石墨烯结晶性好、缺陷少, 非常适 合于基础理论研究 ${ }^{3-5}$ 。根据国际理论化学和应用 化学联合会(IUPAC)的定义, 石墨烯是石墨结构的 单原子碳层, 其结构可类比地描述为具有准无限 尺寸的多环芳烃。然而, 目前作为能源材料使用 的石墨烯材料, 大多是由氧化剥离然后再进行还 原制备而成，由这种方法制备的石墨烯材料被称 为氧化还原石墨烯( $\mathrm{rGO})$ 。氧化还原石墨烯具有制 备简单、可大规模生产、成本低廉等优点，但同 时在制备过程中会不可避免地引入许多缺陷、孔 洞、杂原子以及化学官能团等因素 ${ }^{4}$ 。值得一提的 是, 这些结构上的缺陷和官能团在提高石墨烯基 电极材料的性能方面起着至关重要的作用 ${ }^{6,7}$ 。然 而，这些材料由于制备方法的不同，引入的缺陷 数量、孔洞尺寸以及官能团的类型等也各不相 同, 从而在性能上也表现各异。因此, 如果忽略 这些缺陷因素, 而一味用上述定义来描述这些石 墨烯材料就显得过于简单了; 继续用石墨烯来统 称这些材料, 不仅会引起概念上的混淆, 更无法 将它们的特点清晰地表达出来, 从而给人们, 尤 其是非本专业领域的研究和使用人员造成一定程 度上的混乱。

事实上, 当我们从化学结构的角度分析这些 材料时, 如图 1 所示, 可以看到, 这些所谓的 “石 墨烯”, 其实可以看作是由一个个石墨烯片段通过 聚合物链相互连接而成。这些石墨烯片段构成了 材料的基本结构单元，引入的官能团和缺陷实现

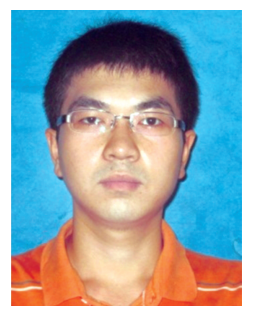

肖志昌，1989 年生。2013 年在首 都师范大学获理学学士学位。 2013 年至今, 在国家纳米科学中 心攻读博士学位。主要研究方向 为基于有机多孔聚合物的结构可 控的富碳纳米材料及其在纳米能 源领域中的应用。

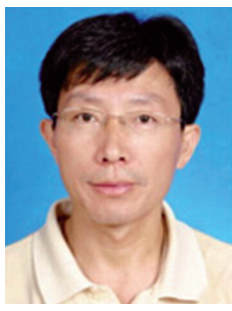

智林杰，1969年生。2000年在中国 科学院煤炭化学研究所获博士学位, 2000-2007年相继在中国科学院化学 研究所和德国马普协会高分子研究所 工作。2007年, 聘为国家纳米科学 中心研究员, 科学院百人计划人选, 博士生导师。主要研究兴趣为富碳纳米材料的构建 与结构控制及其在能源与环境领域的应用。 


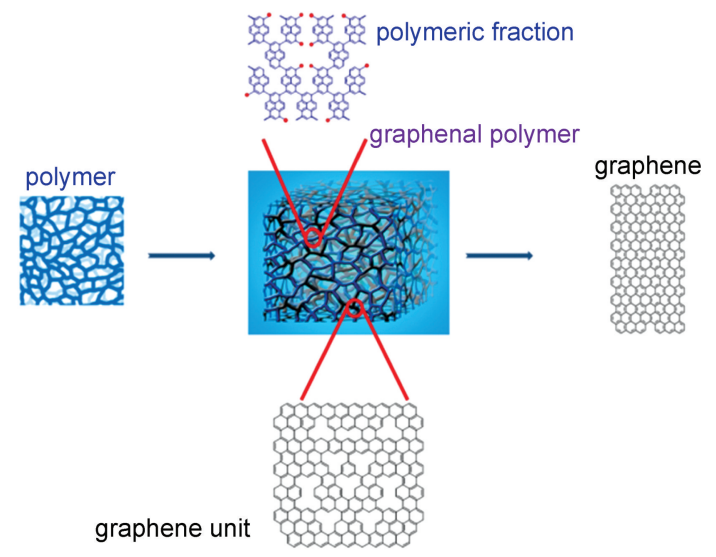

图 1 作为传统聚合物和石墨烯之间过渡态的 石墨烯化聚合物

Fig.1 Graphenal polymers appearing as transitional intermediates lying between the traditional polymers and graphene

了材料的功能化, 而作为桥梁作用的聚合物链使 得其构象变得更加丰富。这些性能既不同于石墨 烯, 也不同于传统的聚合物, 而是表现出了石墨 烯和聚合物的双重特性，因此我们称之为 “石墨 烯化聚合物” 7。之所以引入这一概念, 是希望将 此类材料的研究更多地集中在其化学结构的设计 与构建上, 通过研究其结构和性能的关联关系来 实现其电化学性能的最优化。除了上述的石墨烯 衍生材料，由小分子通过自下而上方法合成的多 孔聚合物网络, 同样具有丰富的孔径、可控的化 学结构和多样的化学官能团。这些聚合物通过特 定的化学交联, 如高温热处理等, 会发生分解和 化学键重排等反应, 使得这些聚合物内部形成许 多类似石墨烯片段的结构, 这些片段同样由聚合 物链所连接。从化学结构角度来看, 这种处理后 的聚合物与上述的石墨烯衍生材料十分相似, 因 此也可以作为 “石墨烯化聚合物” 来研究。由自 下而上方法得到的石墨烯化聚合物, 由于前驱体 结构明确并且来源丰富, 其化学结构更加可控, 也更加多样化, 它们与自上而下方法制备的石墨烯 衍生物一起构成了石墨烯化聚合物的整个谱系, 这 个谱系囊括了由聚合物到石墨烯的过渡阶段, 这类 材料结构丰富、功能可调, 为我们提供了良好的材 料平台来研究其结构与性能之间的关系。

石墨烯化聚合物既具有石墨烯材料优异的导 电性能, 也继承了聚合物丰富的构象与功能, 使 得其具有多样的孔道结构, 有利于形成三维离子 传输通道。因此, 石墨烯化聚合物是一种兼具电
子和离子通道的三维富碳高分子材料, 非常适合 于电化学能源存储与转化性能的研究。本文以此 为切入点, 结合了本课题组和其他研究组在此领 域取得的一些进展, 对两种方法得到的石墨烯化 聚合物进行了较详细的论述和梳理, 探讨了其作 为电化学能源存储和转化器件中的电极材料的应 用, 并对其未来可能的发展方向做了简要讨论。

\section{2 自上而下法制备石墨烯化聚合物}

\section{1 氧化石墨烯造孔方法}

在石墨烯平面上打孔和进行相应的化学改 性，是一种简单有效的制备石墨烯化聚合物的方 法。这种通过造孔方法得到的石墨烯化聚合物, 由于具备特殊的结构与性能, 引起了学术界广泛 的关注和研究 ${ }^{8,9}$ 。在这些方法中, 一种简单有效的 方法是 Zhu等 ${ }^{8}$ 报道的利用 $\mathrm{KOH}$ 活化还原氧化石墨 烯, 从而得到一种石墨烯化聚合物, 即具有多孔 网状结构的石墨烯材料(a-MEGO)(图 2(a))。研究发 现，这种材料具有大量的 $0.6-5 \mathrm{~nm}$ 的孔洞(图 2(b) 和 2(c))、高达 $3100 \mathrm{~m}^{2} \cdot \mathrm{g}^{-1}$ 的超高的 BET 比表面 积、丰富的三维网络结构以及优异的电导率和较 低的氧和氢元素含量。这些独特的结构赋予了这 种石墨烯化聚合物快速的电子和离子传输的性 能, 由此组装的离子液体基超级电容器表现出优 异的性能, 在工作电压为 $3.5 \mathrm{~V}$ 时, $5.7 \mathrm{~A} \cdot \mathrm{g}^{-1}$ 的能 量密度高达 $70 \mathrm{Wh} \cdot \mathrm{kg}^{-1}$ 。此外, $\mathrm{Xu}$ 等 ${ }^{9}$ 报道了一种 利用过氧化氢造孔制备石墨烯化聚合物的方法, 同样表现出优异的电化学性能。他们利用改进的 Hummers 方法得到氧化石墨烯(GO)溶液, 随后用 过氧化氢处理并最终得到三维多孔石墨烯网络结 构材料(图 2(d))。这种石墨烯化聚合物同样具有较 高的 BET 比表面积 $\left(830 \mathrm{~m}^{2} \cdot \mathrm{g}^{-1}\right)$ 、高效的电子和离 子传输通道, 以及很高的堆积密度(面积载量高达 $10 \mathrm{mg} \cdot \mathrm{cm}^{-2}$ ), 用作超级电容器(有机电解液体系)电 极材料表现出 $298 \mathrm{~F} \cdot \mathrm{g}^{-1}$ 的质量比电容和 $212 \mathrm{~F} \cdot \mathrm{cm}^{-3}$ 的体积比电容, 能量密度高达 $35 \mathrm{Wh} \cdot \mathrm{kg}^{-1}$ 和 49 $\mathrm{Wh} \cdot \mathrm{L}^{-1}$, 性能指标已经和目前的铅酸电池相当。

\section{2 石墨烯化聚合物的功能化}

相比精确度较低的氧化石墨烯造孔方法来制 备石墨烯化聚合物，从石墨出发制备氧化石墨 烯, 然后经过还原修饰的方法制备石墨烯化聚合 物可控性更强, 技术方法上也更为成熟, 并且得 到了广泛的应用。这种方法制备的石墨烯化聚合 
(a)
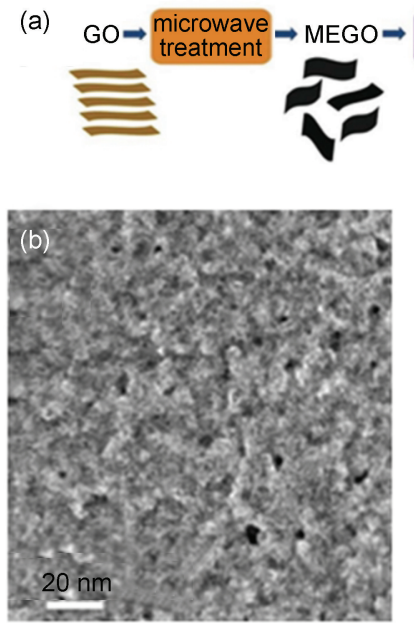
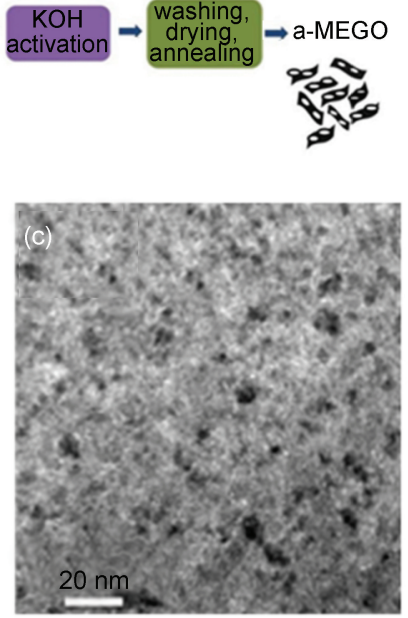

(d)

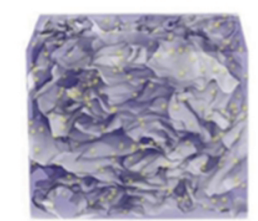

గ compress

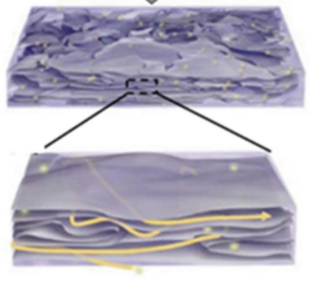

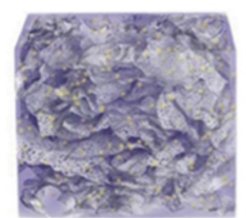

Compress

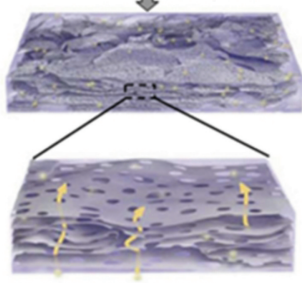

图 2 造孔的还原氧化石墨烯 89

Fig.2 Porous reduced graphene oxide ${ }^{8,9}$

(a) schematic showing the microwave exfoliation/reduction of graphite oxide (GO) and the following chemical activation of microwave exfoliated graphite oxide (MEGO) with $\mathrm{KOH}$ that creates pores while retaining high electrical conductivity; (b) high-resolution scanning electron microscopy

(SEM) image of a different sample region that demonstrates the porous morphology; (c) annular dark field scanning transmission electron microscopy (ADF-STEM) image of the same area as (b), acquired simultaneously. As seen, a-MEGO contains micro- and mesopores with a

distribution of sizes between $\sim 1$ and $\sim 10 \mathrm{~nm}$; (d) schematic illustration of the holey graphene frameworks as an ideal material for EC electrodes

物不仅具有类似石墨烯的二维平面结构，同时还 具有类似有机聚合物的大量活性官能团、杂原子 掺杂和结构缺陷, 赋予其快速的电子和离子传输 的通道, 不仅表现出优异的物理化学稳定性、良 好的电导率，同时也具备了出众的电化学活性 ${ }^{10-12}$ 。

研究表明, 通过官能团修饰的方法能够显著 提高石墨烯化聚合物在超级电容器中的电化学性 能。而通过调节石墨烯的结构与官能团的比例, 可以调节电子、离子的传输通道与提供噟电容的 官能团之间的结合效率, 由此可以深入研究这类 石墨烯化聚合物潜在的性能优势。基于此方法, 我们课题组 ${ }^{11}$ 报道了一种从天然石墨出发, 制备具
有特殊功能结构的石墨烯化聚合物的合成策略。 首先通过改进的 Hummers 方法将天然石墨插层氧 化剥离, 随后结合酸辅助快速热还原的工艺制备 得到具有特定的含氧官能团的石墨烯化聚合物(aFG), 如图 3 所示。通过这种方法所得到的石墨烯 化聚合物, 不但有效地恢复了高电导率的石墨烯 化片段, 而且快速热还原使得材料中保留了大量 的具有赝电容特性的含氧官能团 $(15.16 \%$ 的 $\mathrm{C}-\mathrm{O}$ 结构和 $7.57 \%$ 的 $\mathrm{C}=\mathrm{O}$ 结构), 同时材料本身具有较 高的比表面积 $\left(391 \mathrm{~m}^{2} \cdot \mathrm{g}^{-1}\right)$, 作为超级电容器电极 材料, 表现出非常高的电化学活性, 极高的比容 量和优异的循环性能, 即使在 $10 \mathrm{~A} \cdot \mathrm{g}^{-1}$ 的电流密度

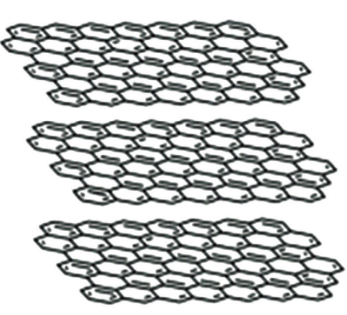

graphite

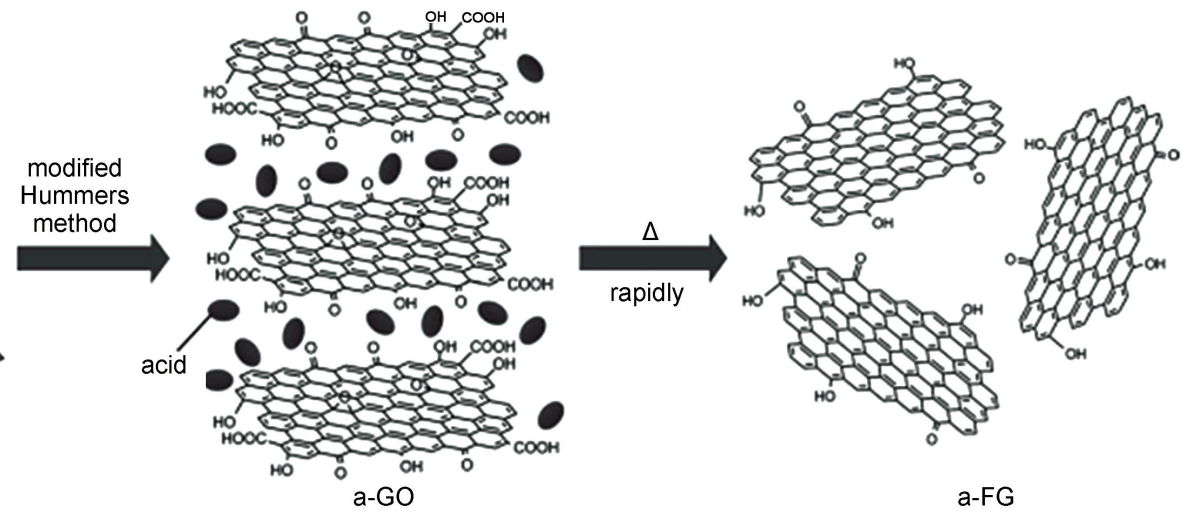

图 3 功能化石墨烯的合成示意图 ${ }^{11}$

Fig.3 Schematic illustration for the synthesis of a-FG ${ }^{11}$ a-GO: acid-incorporated graphene oxide; a-FG: acid-incorporated functionalized graphene 
下, 循环 10000 次后, 比容量仍可保持在 $300 \mathrm{~F}$ $\mathrm{g}^{-1}$ 。同时, 我们 ${ }^{12}$ 成功开发了一种室温下高效还原 固态氧化石墨烯的方法, 进而可以得到具有高导 电性和透光性的柔性石墨烯膜。研究表明, 由改 进的 Hummers 法制备的 GO 溶液均匀喷涂到基底 后, 采用氢气在室温下催化还原便得到我们预期 的功能化的石墨烯化聚合物。在此工作基础上, 我们进一步发展了快速线棒涂敷技术, 在室温下 实现了 GO 膜的高效大面积还原, 得到了兼具高导 电性和高透光率的还原氧化石墨烯膜, 这也是首 次报道的结合线棒涂布和室温还原的方法制备功 能化石墨烯化聚合物膜的工作。

\section{3 石墨烯化聚合物复合材料}

石墨烯化聚合物由于兼具优良的电子和离子 传输通道, 因此也可以作为其他电化学活性物质 的优良载体，从而制备一系列新型的储能材料。 在过去的几年中, 我们课题组 ${ }^{13-20}$ 在这一领域做了 一些初步的探讨。首先, 我们利用液相反应与化 学气相沉积(CVD)相结合的两步组装法成功制备了 二维石墨烯 $/ \mathrm{SnS}_{2}$ 复合材料, 在锂离子电池中表现 出高的可逆容量 $\left(650 \mathrm{mAh} \cdot \mathrm{g}^{-1}\right)$ 和良好的倍率性能 (电流密度 $6400 \mathrm{~mA} \cdot \mathrm{g}^{-1}$ 下可逆容量为 $230 \mathrm{mAh}$. $\left.\mathrm{g}^{-1}\right)$ 。随后我们通过对石墨烯支撑的 $\mathrm{SnO}_{2}$ 进行碳包 覆, 并进一步经过简单的热还原过程, 得到石墨 烯/锡/石墨烯三明治结构。这种独特的石墨烯化聚 合物复合材料中, 石墨烯缓解了充放电过程中金 属锡的体积膨胀, 同时提供了电子和离子传输的 通道, 因此同样表现出优异的锂电循环性能(60次 循环后, 容量保持在 $590 \mathrm{mAh} \cdot \mathrm{g}^{-1}$ 以上)。同时, 我 们首次研究并报道了一种基于石墨烯化聚合物的 锡-核/碳-壳同轴电缆结构。这种结构是通过两步法 实现的: 即氧化石墨烯与氧化锡水热处理后得到 还原氧化石墨烯/氧化锡杂化材料, 进一步 CVD过 程处理得到所述同轴电缆结构。由于独特的形貌 和石墨烯化聚合物复合材料的结构, 该材料表现 出较高的锂存储性能 (50 次循环后容量保持在 630 $\mathrm{mAh} \cdot \mathrm{g}^{-1}$ 以上)。类似地, 我们还报道了一种独特 的 $\mathrm{MoS}_{2}$ 与石墨烯紧密接触的电缆网络结构, 其中 $\mathrm{MoS}_{2}$ 含量可以达到 $90 \%$ 以上。该复合材料在锂离 子电池中表现出 $1150 \mathrm{mAh} \cdot \mathrm{g}^{-1}$ 的比容量, 同时 160 次循环后, 容量保持率接近 $100 \%$, 在 $10 \mathrm{~A} \cdot \mathrm{g}^{-1}$ 的 大倍率测试下, 仍有 $700 \mathrm{mAh} \cdot \mathrm{g}^{-1}$ 的比容量。硅单 质在锂电池负极材料中同样存在体积膨胀、界面
稳定性差等问题。针对这些问题, 我们课题组采 取在硅纳米线上通过 CVD 法包覆石墨烯鞘层得到 SiNW@G 电缆结构, 并进一步与氧化石墨烯复合 并还原得到SiNW@G@RGO 的石墨烯化聚合物复 合材料。相比于未经包覆处理的硅纳米线, 由于 复合材料中, 石墨烯化聚合物缓解了硅工作过程 中的体积膨胀问题, 并且阻止了硅与电解液的直 接接触, 保证了工作过程中的结构和界面的稳定 性; 同时, 丰富的电子和离子传输通道, 也保证 了该材料作为锂电池电极材料时, 能够在 $2.1 \mathrm{~A}$. $\mathrm{g}^{-1}$ 的电流密度下, 100 次循环后容量仍然保持在 $80 \%$ 以上。随后我们利用牛血清素包覆的硅纳米 颗粒与氧化石墨烯通过静电作用组装得到了一种 硅与碳桥相连的定向石墨烯的复合结构(图 4)。同 样地, 石墨烯化聚合物中快速的电子和离子传输通 道, 保证了这种复合材料在锂电池中很高的储锂性 能(电流密度 $2 \mathrm{~A} \cdot \mathrm{g}^{-1}$ 下, 放电比容量高达 1390 $\left.\mathrm{mAh} \cdot \mathrm{g}^{-1}\right)$ 。

\section{3 自下而上法制备石墨烯化聚合物}

\section{1 由多环芳烃构筑的石墨烯化聚合物}

多环芳烃的化学结构主要由 $s p^{2}$ 碳构成, 可以 看作是分子尺度上的石墨烯或石墨烯衍生物，因 此可以作为结构单元来构筑石墨烯化聚合物 ${ }^{21}$; 其中的石墨烯片段可以作为电子传输的介质，同 时这些片段通过聚合物链连接构成三维多孔结 构, 使其成为兼具电子和离子传输通道的富碳高 分子材料。这类材料可由多环芳烃小分子直接热 反应制备而成 ${ }^{22-29}$, 也可以由多环芳烃小分子先通 过化学合成的方法制成芳香共轭聚合物, 然后再 经过热化学交联制备而成 ${ }^{30-34}$ 。最终产物的化学结 构由前驱体的结构和热处理的反应条件共同决 定。因此, 通过选取不同的前驱体和调节热处理 的反应条件, 可以得到结构丰富的一系列石墨烯 化聚合物。

多环芳烃分子通常具有盘状结构, 如最具代 表性的六苯并冦(HBC)及其衍生物 ${ }^{24}$, 如图 5(a) 所 示, 将这些盘状分子注入到多孔氧化铝膜中, 它 们由于 $\pi-\pi$ 相互作用会在氧化铝膜的纳米孔道中 堆叠成柱状结构。经过高温热处理并除去氧化铝 膜后, 就可以得到由石墨烯片层垂直管轴方向规 整排列的碳纳米管(图 5(b))。更有意思的是, 当前 驱体分子不同时, 得到的产物也有形貌上的差 


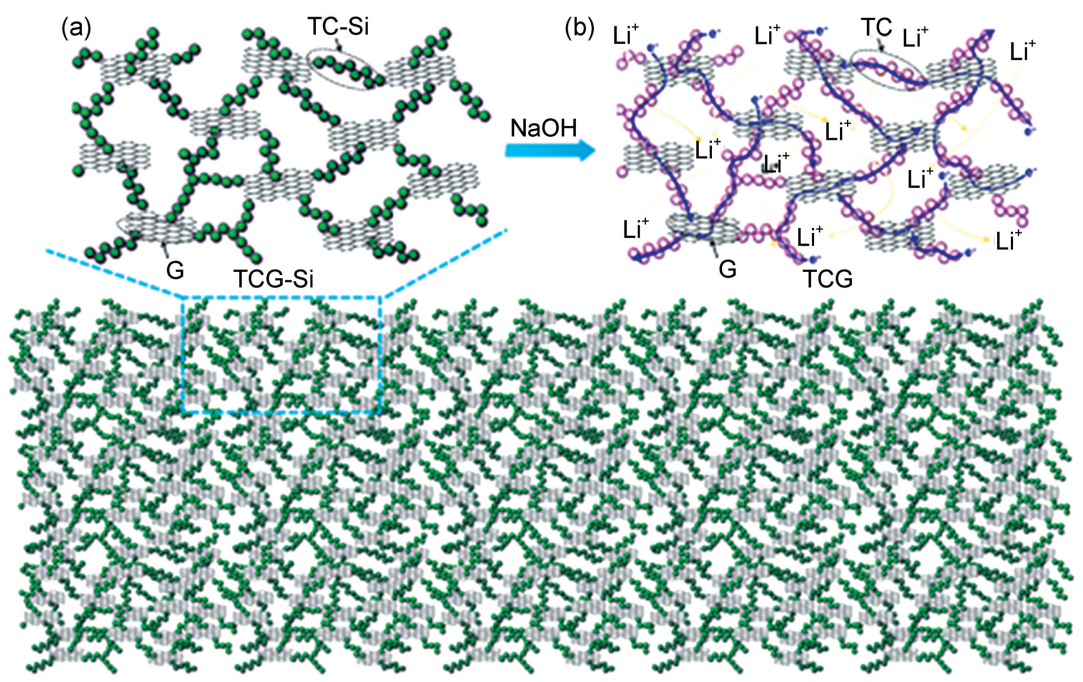

图 4 通过静电作用组装碳-硅电极 19

Fig.4 C-Si electrode design and fabrication ${ }^{19}$

(a) schematic of the configuration of silicon nanoparticle-impregnated assemblies of templated carbon bridged oriented graphene; (b) schematic illustration showing the structure of templated carbon bridged oriented grapheme (TCG) obtained by removing the Si template from the TCG-Si

异 ${ }^{27}$ ，如图 5(c) 所示， HBC-1、HBC-2、HBC-3 分 子均以三维多孔氧化硅为模板, 由 $\mathrm{HBC}-1$ 得到的 碳材料是单分散规整排列的微球, 但是将前驱体 更换为 HBC-2 时, 得到碳球有丰富的孔洞, 并且 相互之间有纳米尺度的通道连接, 而使用 HBC-3 得到的则是中空的碳球。应用此类材料制备的超 级电容器的电极材料, 展现了优异的电化学性 能, 以 $1 \mathrm{~mol} \cdot \mathrm{L}^{-1}$ 硫酸溶液作为电解液, 在 $5 \mathrm{mV}$. $\mathrm{s}^{-1}$ 的扫速下, 比电容可达 $304 \mathrm{~F} \cdot \mathrm{g}^{-1}$, 当扫速增加 到 $100 \mathrm{mV} \cdot \mathrm{s}^{-1}$ 时, 仍可得到 $243 \mathrm{~F} \cdot \mathrm{g}^{-1}$ 的比电容。

除了采用多环芳烃小分子直接热交联得到目 标产物, 石墨烯化聚合物的制备往往选取共轭微
孔芳香聚合物 ${ }^{35}$ 作为前驱体，这类聚合物具有聚苯 撑结构, 在热处理过程中有利于石墨烯片段的形 成, 同时具有丰富的孔结构, 在电化学应用中可以 作为电解液离子流通的通道。Cooper 课题组 ${ }^{36}$ 利用 苯炔单体和卤代芳烃单体的 Sonogashira-Hagihara 交联反应合成了一系列微孔共轭聚合物, 通过选 取不同的分子结构的单体就可以调控最终产物的 孔结构。他们运用分子模拟的方法证实了链接分 子的弯曲振动在孔结构的形成中所起的作用(图 6 (a))。除了上述的 Sonogashira-Hagihara 交联反应, 还有一系列的反应如付克烷基化反应 ${ }^{37,38}$ 、Suzuki 交联反应 ${ }^{39} 、$ Scholl 反应 ${ }^{40} 、$ Knitting 反应 ${ }^{41}$ 等, 均 (a)

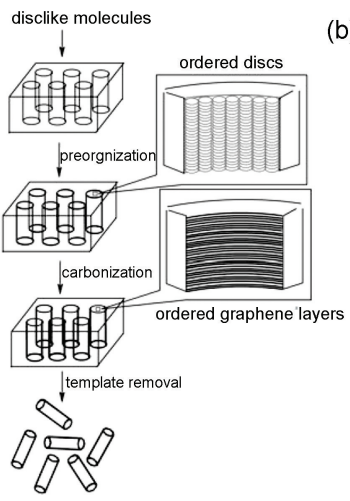

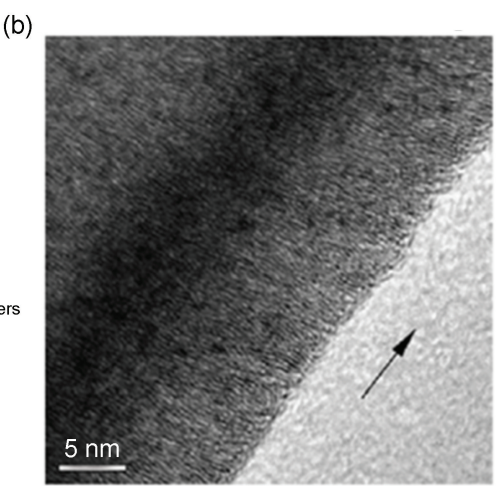

(c)
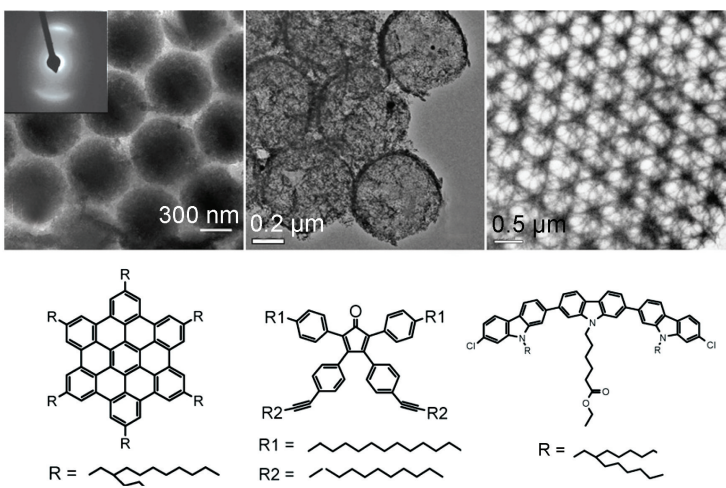

图 5 多环芳烃构筑的石墨烯化聚合物 ${ }^{21,24,27}$

Fig.5 Polycyclic aromatic hydrocarbons as precursors for graphenal polymers formation ${ }^{21,24,27}$

(a) the formation of carbon nanotubes with controlled orientation of graphene layers; (b) high-resolution transmission electron microscopy (HR-TEM) image of as-prepared carbon nanotubes with highly ordered graphene layers oriented perpendicularly to the tube axis. The arrow indicates the tube axis. (c) The structure of the carbonaceous product is closely related to the structure of the precursors. 

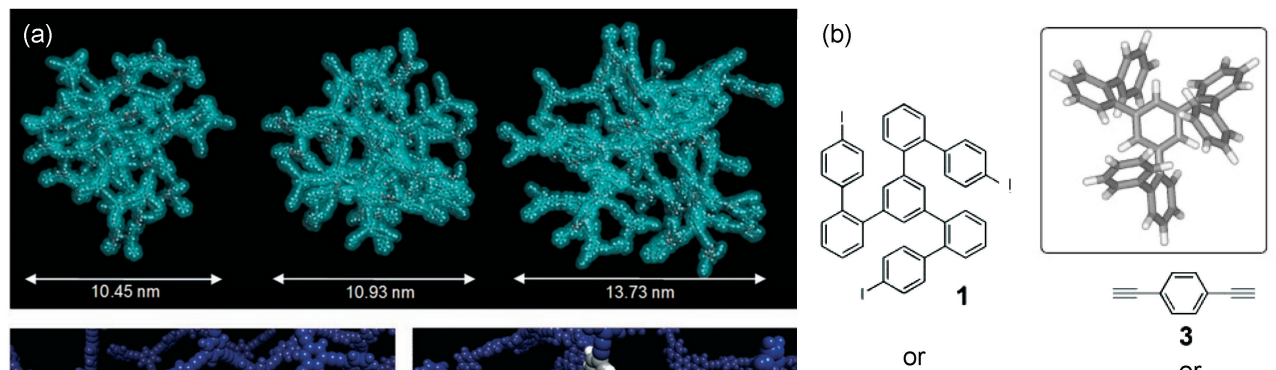

CPN1 (1+3)
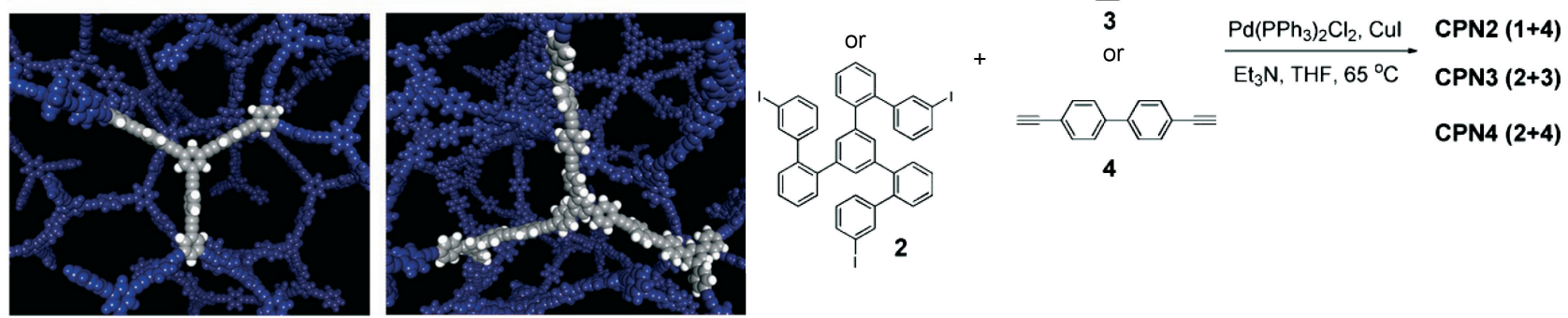

图 6 共轭微孔聚合物构筑的石墨烯化聚合物 ${ }^{30,36}$

Fig.6 Conjugated microporous polymers as precursors for graphenal polymers formation ${ }^{30,36}$ (a) atomistic simulations and microscopy for polyyne networks; (b) synthesis of cross-linked conjugated polymers

可以用来合成共轭多孔聚合物，这为石墨烯化聚 合物的制备提供了丰富的前驱体。如图 6(b)所示, 我们使用了不同构象的碘代芳烃 1、2 和苯炔类单 体 3、4 得到了微孔碳纳米线和碳纳米管两种形 貌, 比表面积达 $900 \mathrm{~m}^{2} \cdot \mathrm{g}^{-1}$ 以上, 是电化学电容器 的理想电极材料 ${ }^{30}$ 。

\section{2 由共价/金属有机骨架衍生的石墨烯化聚合物}

共价/金属有机骨架是通过共价键和金属配位 键连接的有规整孔结构的有机物 ${ }^{42-45}$, 是制备石墨

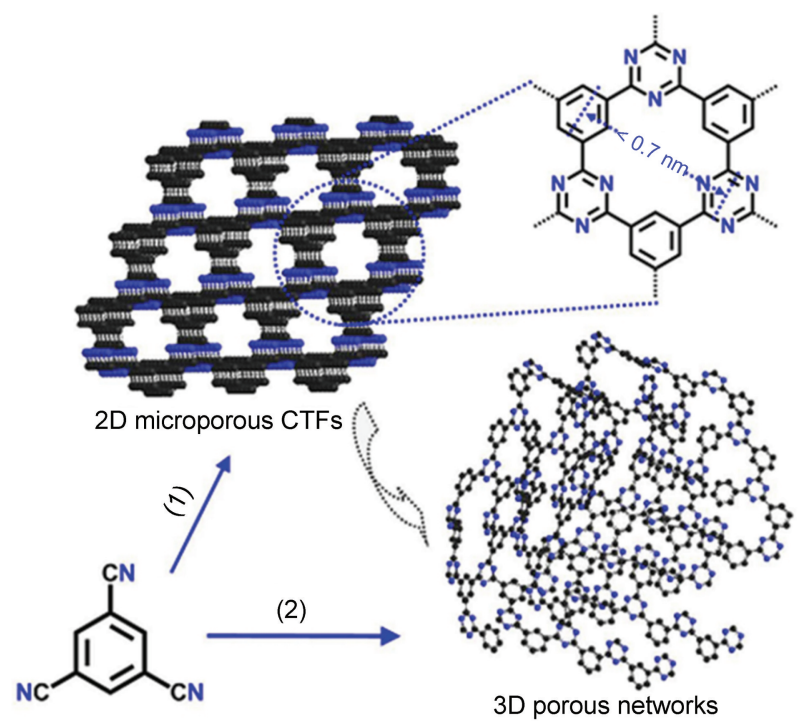

图 7 氭基二维有序多孔聚合物演变成氮掺杂三维多孔 石墨烯化聚合物 ${ }^{50}$

Fig.7 Structural evolution of 2D microporous covalent triazine-based framework toward 3D nitrogen-containing porous graphenal polymer ${ }^{50}$

CTFs: covalent triazine-based frameworks
烯化聚合物的一种理想前驱体。这类有机骨架往 往含有均匀掺杂的杂原子、长程有序的孔结构、 高比表面积等。这些特征有利于电解液离子的流 通, 但这些材料导电性很差。不过, 在经过高温 处理后, 得到的产物能够在保留上述特征的同 时, 大大提高其导电性, 从而成为理想的电化学 电极材料 ${ }^{46}$ 。其中, 共价三嗪环骨架 (CTF) 由于其 均匀的氮掺杂、高比表面积和简易的制备方法等 优点得到了广泛的研究 ${ }^{42,46-50}$ 。如图 7所示, CTF 是 使用芳香腈化合物为单体, 在 $400{ }^{\circ} \mathrm{C}$ 的氯化锌熔 融盐中, 通过氧基的环三聚反应制备而成, 是一 种二维有序孔结构的有机物。当升高合成温度 时, CTF 由二维有序微孔结构转变为三维介孔碳 材料。我们课题组 ${ }^{50}$ 首次将这种材料应用于超级电 容器的电极材料, 得到良好的电化学性能, 在 1 $\mathrm{mol} \cdot \mathrm{L}^{-1}$ 硫酸电解液中, 在 $0.2 \mathrm{~A} \cdot \mathrm{g}^{-1}$ 的电流密度下 得到 $220 \mathrm{~F} \cdot \mathrm{g}^{-1}$ 的比电容。此外, 这种材料还是一 种理想的模型材料, 可以用来研究电极材料的介 电常数和其比电容间的关系。

\section{4 结 论}

本文总结了近年来越来越引起关注的一系列 含有石墨烯片段结构的聚合物及其作为电化学电 极材料在能源存储和转化器件中应用的研究工 作。为了便于对这类功能材料进行系统的研究, 基于这类聚合物的化学结构的特点, 我们将之统 称为石墨烯化聚合物。石墨烯化聚合物可由两种 合成策略获得：(1) 自上而下法, 即以石墨为原 
料, 通过掺杂、打孔、剥离、功能化等方式制备 得到; (2) 自下而上法, 即由多环芳烃单体或共价/ 金属有机骨架衍生制备得到。自上而下法有可能 实现石墨烯化聚合物的大批量低成本制备, 在实 际生产中有着潜在的应用前景; 而自下而上法则 可以通过改变单体的种类和合成条件来更精确地 调控石墨烯化聚合物的结构与功能, 从而为研究 这类材料的结构与性能的关系提供了较理想的模 型平台。

如本文所述，石墨烯化聚合物表现出良好的 导电性能和丰富的孔结构, 使其拥有优异的电子 导通能力的同时, 也有利于电解液离子的快速传 输, 这种兼具电子和离子传输通道的三维结构使 其在能源存储和转化中展现出了广阔的应用前 景。近年来，针对石墨烯化聚合物的结构特性及 其与电化学性能的关系的研究越来越引起学术界 的重视, 并且在能源材料应用领域展示出许多有 吸引力的实验结果。更重要的是, 石墨烯化聚合 物具有独特的结构与性能特性，既不同于传统的 聚合物, 也不同于石墨烯, 无法用传统的高分子 和/或碳材料研究方法来系统深入研究这类材料。 建立合理可行的石墨烯化聚合物研究方法学就显 得非常重要。我们也期望各领域的同行能一起参 与讨论, 为更深入高效地研究这类材料提供更多 的指导和建议。相信随着人们对这类材料研究的 逐渐深入，石墨烯化聚合物必将在能源存储和转 化领域展现出其独特的结构与性能优势。

\section{References}

(1) Winter, M.; Brodd, R. J. Chem. Rev. 2004, 104, 4245. doi: $10.1021 / \mathrm{cr} 020730 \mathrm{k}$

(2) Bonaccorso, F.; Colombo, L.; Yu, G.; Stoller, M.; Tozzini, V.; Ferrari, A. C.; Ruoff, R. S.; Pellegrini, V. Science 2015, 347, 41. doi: 10.1126/science. 1246501

(3) Luo, B.; Zhi, L. Energy Environ. Sci. 2015, 8, 456. doi: 10.1039/ c4ee $02578 \mathrm{~d}$

(4) Ambrosi, A.; Chua, C. K.; Bonanni, A.; Pumera, M. Chem. Rev. 2014, 114, 7150. doi: 10.1021/cr500023c

(5) Liang, M.; Zhi, L. J. Mater. Chem. 2009, 19, 5871. doi: 10.1039/ b901551e

(6) Liang, M.; Luo, B.; Zhi, L. Int. J. Energy Res. 2009, 33, 1161. doi: $10.1002 /$ er. 1598

(7) Li, X.; Song, Q.; Hao, L.; Zhi, L. Small 2014, 10, 2122. doi: 10.1002/smll.201303717

(8) Zhu, Y.; Murali, S.; Stoller, M. D.; Ganesh, K. J.; Ruoff, R. S. Science 2011, 332, 1537. doi: 10.1126/science.1200770
(9) Xu, Y.; Lin, Z.; Zhong, X.; Huang, X.; Weiss, N. O.; Huang, Y.; Duan, X. Nat. Commun. 2014, 5, 4554. doi: 10.1038/ ncomms5554

(10) Fan, Z.; Yan, J.; Zhi, L.; Zhang, Q.; Wei, T.; Feng, J.; Zhang, M.; Qian, W.; Wei, F. Adv. Mater. 2010, 22, 3723. doi: 10.1002/ adma.201001029

(11) Fang, Y.; Luo, B.; Jia, Y.; Li, X.; Wang, B.; Song, Q.; Kang, F.; Zhi, L. Adv. Mater. 2012, 24, 6348. doi: 10.1002/ adma.201202774

(12) Liang, M.; Wang, J.; Luo, B.; Qiu, T.; Zhi, L. Small 2012, 8 , 1180. doi: 10.1002 / smll. 201101968

(13) Wang, B.; Li, X.; Zhang, X.; Luo, B.; Jin, M.; Liang, M.; Dayeh, S. A.; Picraux, S. T.; Zhi, L. ACS Nano 2013, 7, 1437. doi: $10.1021 / \mathrm{nn} 3052023$

(14) Luo, B.; Wang, B.; Li, X.; Jia, Y.; Liang, M.; Zhi, L. Adv. Mater 2012, 24, 3538. doi: 10.1002/adma.201201173

(15) Luo, B.; Wang, B.; Liang, M.; Ning, J.; Li, X.; Zhi, L. Adv. Mater. 2012, 24, 1405. doi: 10.1002/adma.201104362

(16) Wang, J.; Liang, M.; Fang, Y.; Qiu, T.; Zhang, J.; Zhi, L. $A d v$. Mater. 2012, 24, 2874. doi: 10.1002/adma.201200055

(17) Kong, D.; He, H.; Song, Q.; Wang, B.; Lv, W.; Yang, Q. H.; Zhi, L. Energy Environ. Sci. 2014, 7, 3320. doi: 10.1039/C4ee02211d

(18) Luo, B.; Fang, Y.; Wang, B.; Zhou, J.; Song, H.; Zhi, L. Energy Environ. Sci. 2012, 5, 5226. doi: 10.1039/c1ee02800f

(19) Zhou, M.; Li, X.; Wang, B.; Zhang, Y.; Ning, J.; Xiao, Z.; Zhang, X.; Chang, Y.; Zhi, L. Nano Lett. 2015, 15, 6222. doi: 10.1021/acs.nanolett.5b02697

(20) Wang, B.; Li, X.; Luo, B.; Yang, J.; Wang, X.; Song, Q.; Chen, S.; Zhi, L. Small 2013, 9, 2399. doi: 10.1002/smll.201300692

(21) Zhi, L.; Muellen, K. J. Mater. Chem. 2008, 18, 1472. doi: $10.1039 / \mathrm{b} 717585 \mathrm{j}$

(22) Zhi, L.; Wu, J.; Li, J.; Stepputat, M.; Kolb, U.; Müllen, K. $A d v$. Mater. 2005, 17, 1492. doi: 10.1002/adma.200500290

(23) Zhi, L. J.; Gorelik, T.; Friedlein, R.; Wu, J. S.; Kolb, U.; Salaneck, W. R.; Mullen, K. Small 2005, 1, 798. doi: 10.1002/ sml1.200500150

(24) Zhi, L. J.; Wu, J. S.; Li, J. X.; Kolb, U.; Mullen, K. Angew. Chem. Int. Ed. 2005, 44, 2120. doi: 10.1002/anie.200460986

(25) Cui, G.; Zhi, L.; Thomas, A.; Kolb, U.; Lieberwirth, I.; Muellen, K. Angew. Chem. Int. Ed. 2007, 46, 3464. doi: 10.1002/ anie. 200700077

(26) Wu, D.; Zhi, L.; Bodwell, G. J.; Cui, G.; Tsao, N.; Muellen, K. Angew. Chem. Int. Ed. 2007, 46, 5417. doi: 10.1002/ anie. 200700515

(27) Zhi, L.; Wang, J.; Cui, G.; Kastler, M.; Schmaltz, B.; Kolb, U.; Jonas, U.; Muellen, K. Adv. Mater. 2007, 19, 1849. doi: 10.1002/ adma.200602365

(28) Liu, R.; Wu, D.; Feng, X.; Mullen, K. Angew. Chem. Int. Ed. 2010, 49, 2565. doi: 10.1002/anie.200907289

(29) Yang, S.; Feng, X.; Zhi, L.; Cao, Q.; Maier, J.; Muellen, K. Adv. Mater. 2010, 22, 838. doi: 10.1002/adma.200902795 
(30) Feng, X.; Liang, Y.; Zhi, L.; Thomas, A.; Wu, D.; Lieberwirth, I.; Kolb, U.; Muellen, K. Adv. Funct. Mater. 2009, 19, 2125. doi: 10.1002/adfm.200900264

(31) Liang, Y.; Feng, X.; Zhi, L.; Kolb, U.; Muellen, K. Chem. Commun. 2009, 809. doi: 10.1039/b819202b

(32) Vilela, F.; Zhang, K.; Antonietti, M. Energy Environ. Sci. 2012, 5, 7819. doi: 10.1039/ c2ee22002d

(33) Song, Q.; Jia, Y.; Luo, B.; He, H.; Zhi, L. Small 2013, 9, 2460. doi: 10.1002/ smll. 201301194

(34) Zhuang, X.; Gehrig, D.; Forler, N.; Liang, H.; Wagner, M.; Hansen, M. R.; Laquai, F.; Zhang, F.; Feng, X. Adv. Mater. 2015, 27, 3789. doi: 10.1002/adma.201501786

(35) Xu, C.; Hedin, N. Mater. Today 2014, 17, 397. doi: 10.1016/j. mattod.2014.05.007

(36) Jiang, J. X.; Su, F.; Trewin, A.; Wood, C. D.; Campbell, N. L.; Niu, H.; Dickinson, C.; Ganin, A. Y.; Rosseinsky, M. J.; Khimyak, Y. Z.; Cooper, A. I. Angew. Chem. Int. Ed. 2007, 46, 8574. doi: 10.1002/anie.200701595

(37) Wood, C. D.; Tan, B.; Trewin, A.; Niu, H.; Bradshaw, D.; Rosseinsky, M. J.; Khimyak, Y. Z.; Campbell, N. L.; Kirk, R.; Stockel, E.; Cooper, A. I. Chem. Mater. 2007, 19, 2034. doi: $10.1021 / \mathrm{cm} 070356 \mathrm{a}$

(38) Wood, C. D.; Tan, B.; Trewin, A.; Su, F.; Rosseinsky, M. J.; Bradshaw, D.; Sun, Y.; Zhou, L.; Cooper, A. I. Adv. Mater. 2008, 20, 1916. doi: 10.1002/adma.200702397

(39) Chen, L.; Honsho, Y.; Seki, S.; Jiang, D. J. Am. Chem. Soc. 2010, 132, 6742. doi: 10.1021/ja100327h

(40) Li, B.; Guan, Z.; Yang, X.; Wang, W. D.; Wang, W.; Hussain, I.;
Song, K.; Tan, B.; Li, T. J. Mater. Chem. A 2014, 2, 11930. doi: $10.1039 / \mathrm{c} 4 \mathrm{ta} 01081 \mathrm{~g}$

(41) Luo, Y.; Li, B.; Wang, W.; Wu, K.; Tan, B. Adv. Mater. 2012, 24, 5703. doi: 10.1002/ adma.201202447

(42) Kuhn, P.; Antonietti, M.; Thomas, A. Angew. Chem. Int. Ed. 2008, 47, 3450. doi: 10.1002/anie. 200705710

(43) Kou, Y.; Xu, Y.; Guo, Z.; Jiang, D. Angew. Chem. Int. Ed. 2011, 50, 8753. doi: 10.1002 /anie. 201103493

(44) Chen, Y. Z.; Wang, C.; Wu, Z. Y.; Xiong, Y.; Xu, Q.; Yu, S. H.; Jiang, H. L. Adv. Mater. 2015, 27, 5010. doi: 10.1002/ adma. 201502315

(45) Sakaushi, K.; Antonietti, M. Accounts Chem. Res. 2015, 48, 1591. doi: 10.1021/acs.accounts.5b00010

(46) Kuhn, P.; Forget, A.; Su, D.; Thomas, A.; Antonietti, M. J. Am. Chem. Soc. 2008, 130, 13333. doi: 10.1021/ja803708s

(47) Hao, L.; Luo, B.; Li, X.; Jin, M.; Fang, Y.; Tang, Z.; Jia, Y.; Liang, M.; Thomas, A.; Yang, J.; Zhi, L. Energy Environ. Sci. 2012, 5, 9747. doi: 10.1039/c2ee22814a

(48) Zhu, X.; Tian, C.; Mahurin, S. M.; Chai, S. H.; Wang, C.; Brown, S.; Veith, G. M.; Luo, H.; Liu, H.; Dai, S. J. Am. Chem. Soc. 2012, 134, 10478. doi: 10.1021/ja304879c

(49) Su, Y.; Liu, Y.; Liu, P.; Wu, D.; Zhuang, X.; Zhang, F.; Feng, X. Angew. Chem. Int. Ed. 2015, 54, 1812. doi: 10.1002/ anie. 201410154

(50) Hao, L.; Ning, J.; Luo, B.; Wang, B.; Zhang, Y.; Tang, Z.; Yang, J.; Thomas, A.; Zhi, L. J. Am. Chem. Soc. 2015, 137, 219. doi: $10.1021 / j a 508693 y$ 